\title{
Multiple steps prediction with nonlinear ARX models
}

\author{
Qinghua Zhang, Lennart Ljung \\ Division of Automatic Control \\ E-mail: zhang@irisa.fr, ljung@isy.liu.se
}

14th June 2007

Report no.: LiTH-ISY-R-2793

Accepted for publication in Proc. NOLCOS 2004 - IFAC Symposium on Nonlinear Control Systems, Stuttgardt

Address:

Department of Electrical Engineering

Linköpings universitet

SE-581 83 Linköping, Sweden

WWW: http://WwW. control.isy.liu.se

AUTOMATIC CONTROL

REGLERTEKNIK

LINKÖPINGS UNIVERSITET

Technical reports from the Automatic Control group in Linköping are available from http://www. control.isy.liu.se/publications. 


\begin{abstract}
NLARX (NonLinear AutoRegresive with eXogenous inputs) models are frequently used in black-box nonlinear system identification. Though it is easy to make one step ahead prediction with such models, multiple steps prediction ids far from trivial. The main difficulty is that in general there is no easy way to compute the mathemaical expectation of an output conditioned by past measurements. An optimal solution would require intensive numerical computations related to nonlinear filtering. The purpose of this paper is to investigate simple non-optimal prediction merhods. It is shown that caution must be paid when using such methods, since the prediction behaviors may be radically different, depending on some detailed choices.
\end{abstract}

Keywords: identification 


\title{
MULTIPLE STEPS PREDICTION WITH NONLINEAR ARX MODELS
}

\author{
Qinghua Zhang* Lennart Ljung**
}

\author{
* IRISA-INRIA, Campus de Beaulieu, 35042 Rennes Cedex, France \\ Email: zhang@irisa.fr \\ ** Div. of Automatic Control, Linköping University, \\ SE-58183 Linköping, Sweden.Email: ljung@isy.liu.se
}

\begin{abstract}
NLARX (NonLinear AutoRegressive with eXogenous inputs) models are frequently used in black-box nonlinear system identification. Though it is easy to make one step ahead prediction with such models, multiple steps prediction is far from trivial. The main difficulty is that in general there is no easy way to compute the mathematical expectation of an output conditioned by past measurements. An optimal solution would require intensive numerical computations related to nonlinear filtering. The purpose of this paper is to investigate simple non optimal prediction methods. It is shown that cautions must be paid when using such methods, since their prediction behaviors may be radically different, depending on some detailed choice.
\end{abstract}

Keywords: nonlinear system identification, NLARX model, multiple steps prediction, simulation.

\section{INTRODUCTION}

In the field of classical linear system identification, ARX (AutoRegressive with eXogenous inputs), ARMAX (AutoRegressive and Moving Average with eXogenous inputs) and OE (Output Error) models have been extensively studied. Let us just cite a few examples out of the very vast literature on this topic: (Goodwin and Payne, 1977; Söderström and Stoica, 1989; Walter and Pronzato, 1997; Ljung, 1999). For nonlinear system identification, researches have been mainly focused on NonLinear ARX (or NLARX) models, notably since the emergence of artificial neural networks applied to nonlinear system identification (Narendra and Parthasarathy, 1990; Levin and Narendra, 1995; Sjöberg et al., 1995; Juditsky et al., 1995; Principe et al., 1998; Chen et al., 1999). The purpose of this paper is to investigate some issues on the way NLARX models are used for multiple steps prediction.

NLARX models can be used for various purposes (control, simulation, behavior analysis, monitoring, etc.). When one step ahead prediction is needed in such applications, the predictor can be trivially derived from an NLARX model. In some applications, multiple steps prediction of the identified system is required. It turns out that multiple steps prediction is a problem far less trivial. In the linear case, when the modeling error is simply assumed to be a white noise, the $k$ steps ahead prediction of the output at the time instant $t$ can be made by computing its mathematical expectation conditioned by the measured outputs up to the time instant $t-k$. If, moreover, the modeling error is assumed Gaussian, then the full conditional distribution of the predicted output can be computed based on the Kalman theory. In the nonlinear case, however, the problem becomes much more complicated. In general, the conditional mean of the output to be predicted cannot be simply computed without estimating its full conditional distribution. In principle, this problem is related to nonlinear filtering theory (Jazwinski, 1970). However, the implementation of such solutions requires intensive numerical computations. Even with the recently developed particle filtering methods (Doucet et al., 2001), the necessary nu- 
merical computations can rarely be accomplished in real time for most applications. For engineering practices, simple methods for multiple steps prediction are often required. It is thus of practical importance to investigate simple non optimal multiple steps prediction methods for nonlinear systems. This is exactly the purpose of this paper for NLARX models.

Remark that the multiple steps predictions considered in this paper are based on an NLARX model estimated through the minimization of one-step prediction error criterion. This is typically the approach used in Model Predictive Control (MPC). However, some authors suggest to estimate different models for different prediction horizons (Di Palma and Magni, 2004).

We will investigate in this paper two simple methods for multiple steps prediction derived from iterations of the NLARX model by neglecting some modeling errors. These apparently natural methods are not new and differ only in some detail. However, as will be shown in the paper, the slight difference leads to completely different behaviors for multiple steps prediction. Very often, one of the methods gives badly oscillatory results, whereas the other one exhibits a more reasonable behavior.

For presentation simplicity, only single input single output (SISO) systems are considered in this paper.

\section{METHOD 1}

In this section we examine a first apparently natural multiple steps prediction method

\subsection{NLARX model and one step ahead prediction}

Let let $u(t)$ and $y(t)$ be respectively the measured system input and output at discrete time instants $t=1,2,3, \ldots$, then a NLARX model has the form

$$
\begin{gathered}
y(t)=f\left[y(t-1), \ldots, y\left(t-n_{a}\right), u\left(t-n_{k}\right), \ldots,\right. \\
\left.u\left(t-n_{k}-n_{b}+1\right)\right]+e(t)
\end{gathered}
$$

where the integers $n_{a}, n_{b}$ are the numbers of past outputs and inputs involved in the model, $n_{k}$ is the pure input delay, $f$ is some nonlinear function (typically implemented by an artificial neural network), and $e(t)$ represents the modeling error. Such a model is typically estimated by minimizing some criterion based on the error sequence $e(t)$ with respect to some parametrization vector of the nonlinear function $f$.

With such a NLARX model, a prediction of the system output at time instant $t$ from past mea- sured outputs is obtained by simply omitting the error $e(t)$ :

$$
\begin{gathered}
\hat{y}(t)=f\left[y(t-1), \ldots, y\left(t-n_{a}\right), u\left(t-n_{k}\right), \ldots,\right. \\
\left.u\left(t-n_{k}-n_{b}+1\right)\right]
\end{gathered}
$$

If the error sequence $e(t)$ is a white noise independent of past measurements, then this prediction coincides with the conditional mean:

$$
\begin{aligned}
\hat{y}(t)=\mathbf{E}\left[y(t) \mid y(t-1), \ldots, y\left(t-n_{a}\right),\right. \\
\\
\left.u\left(t-n_{k}\right), \ldots, u\left(t-n_{k}-n_{b}+1\right)\right]
\end{aligned}
$$

It is considered as a one step ahead prediction, because $\hat{y}(t)$ is computed from measured outputs up to the time instant $t-1$.

\subsection{Multiple steps prediction}

Based on the one step prediction formula (2), an apparently natural multiple steps prediction is derived by recursively applying the same prediction formula as follows. For $k=1,2,3, \ldots$, let $\hat{y}^{(k)}(t)$ denote the $k$ step prediction of the output $y(t)$. The one step prediction is simply $\hat{y}^{(1)}(t) \triangleq \hat{y}(t)$ with $\hat{y}(t)$ as defined in (2). Then for $k=2,3,4 \ldots$, the $k$ step prediction is recursively computed by

$$
\begin{array}{r}
\hat{y}^{(k)}(t)=f\left[\hat{y}^{(k-1)}(t-1), \ldots, \hat{y}^{(k-1)}\left(t-n_{a}\right),\right. \\
\left.u\left(t-n_{k}\right), \ldots, u\left(t-n_{k}-n_{b}+1\right)\right]
\end{array}
$$

Notice that the sequence $\hat{y}^{(1)}(t)$ (for $t=1,2,3, \ldots$ ) must be first computed before $\hat{y}^{(2)}(t)$ is computed, then $\hat{y}^{(3)}(t)$ is computed, and so on. This prediction formula is particularly attractive for batch processing of data in Matlab, since it can be implemented efficiently with a loop over the steps $k$ instead of a loop over the data samples.

The value $\hat{y}^{(k)}(t)$ computed with the recurrent formula (3) can be viewed as a $k$ step ahead prediction of $y(t)$, since it is computed from measured outputs up to the time instant $t-k$. This method seems natural, since it repeatedly applies the one step prediction formula, each time for a further step prediction. However, this method typically leads to badly oscillatory results, as illustrated by the following example.

The considered example is a liquid-saturated steam heat exchanger for which a description and the available data can be found in the data base (De Moor, 1996). The system has an input, the liquid flow rate, and an output, the outlet liquid temperature. The 4000 samples of input-output data downloaded from the data base are plotted in figure 1. The data from sample 220 to 2000 are used for the estimation of a NLARX model with $n_{a}=3, n_{b}=3, n_{k}=1$ and $f$ implemented as a single hidden layer neural network of 2 sigmoid neurons.

The one step prediction with the estimated NLARX model is then tested over the entire 4000 

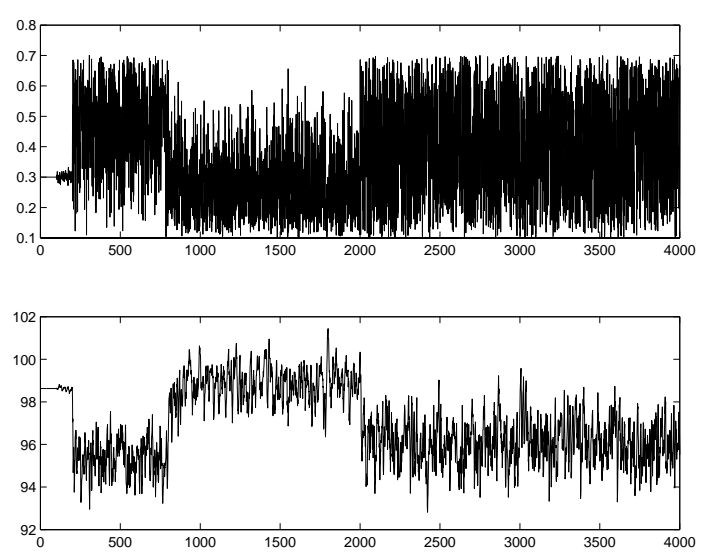

Figure 1. Input (top) and output (bottom) data of the liquid-saturated steam heat exchanger

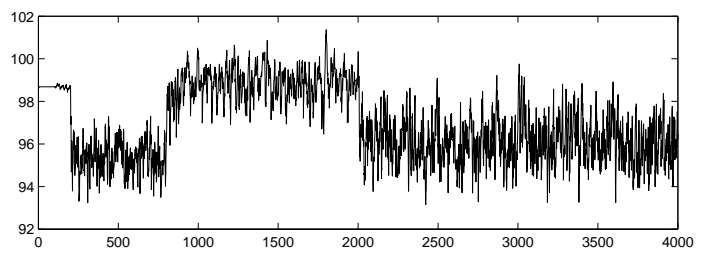

Figure 2. One step ahead prediction for the liquidsaturated steam heat exchanger data

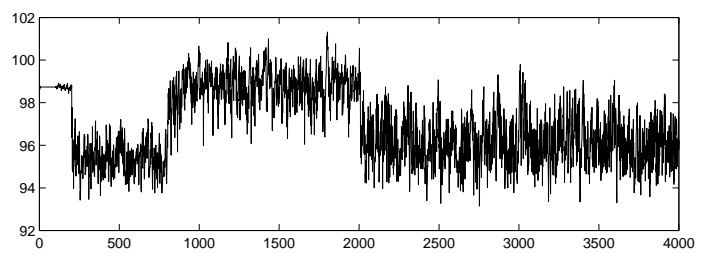

Figure 3. Two steps ahead prediction for the liquid-saturated steam heat exchanger data

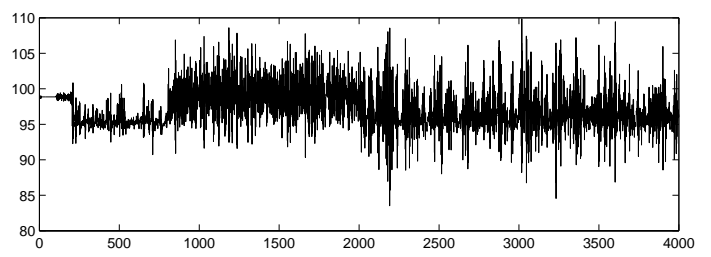

Figure 4. Five steps ahead prediction for the liquid-saturated steam heat exchanger data

data samples, as plotted in figure 2. As expected, the one step prediction is similar to the measured output of figure 1 , so is the two steps prediction shown in figure 3 . However, the degradation of the five steps prediction plotted in figure 4 is clearly noticeable. The result of the ten steps prediction given in figure 5 is completely meaningless.

One may think that this bad prediction behavior is due to the instability of the NLARX model. Since it is difficult to analytically study the stability of such a nonlinear model, let us try to get some idea by simulation. The simulated output $z(t)$ is computed with the estimated model as follows. First choose the initial values

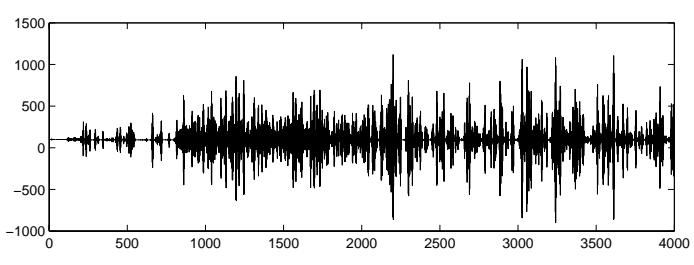

Figure 5. Ten steps ahead prediction for the liquidsaturated steam heat exchanger data

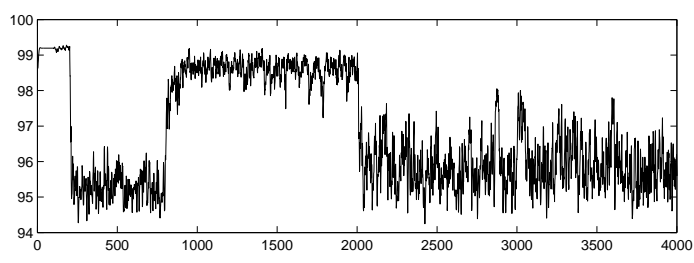

Figure 6. Simulated output for the liquidsaturated steam heat exchanger example

$z(1), z(2), \ldots, z\left(n_{a}\right)$, then $z(t)$ is recursively computed for $t>n_{a}$ :

$$
\begin{aligned}
z(t)=f\left[z(t-1), \ldots, z\left(t-n_{a}\right),\right. & \\
& \left.u\left(t-n_{k}\right), \ldots, u\left(t-n_{k}-n_{b}+1\right)\right]
\end{aligned}
$$

The simulation is made with the same input data $u(t)$ as above. Since the input $u(t)$ is independent of the simulated output $z(t)$, if the recurrent equation (4) is instable, typically the simulated $z(t)$ will diverge.

The simulated output is illustrated in figure 6 . It can be noticed that the simulated output is much more similar to the measured output than the five and ten steps predictions. The simulation result supports the guess that the estimated NLARX model is stable. Anyway it is surprising that the five and ten steps predictions are much worse than the result of the simulation which can be viewed as infinite steps prediction.

\subsection{What is wrong?}

One may question if this surprisingly bad behavior of multiple steps prediction is caused by the nonlinearity of the estimated model. It turns out that this problem is not specific to nonlinear systems. If the multiple steps prediction formula (3) is applied to linear systems, the same behavior can also be observed. For example, consider the linear AR model

$$
y(t)=a_{1} y(t-1)+a_{2} y(t-2)+e(t)
$$

Assume that model parameters has been estimated somehow, and a data set $y(t)$ for $t=$ $1,2, \ldots, N$ is given. The prediction formula $(3)$ is then applied to make multiple steps predictions. For simplicity, assume $y(1)=y(2)=0$. Define the notations 


$$
Y=\left[\begin{array}{c}
y_{1} \\
\vdots \\
y_{N}
\end{array}\right] \quad \hat{Y}^{(k)}=\left[\begin{array}{c}
\hat{y}_{1}^{(k)} \\
\vdots \\
\hat{y}_{N}^{(k)}
\end{array}\right]
$$

the recurrent equation (3) leads, for this particular linear example, to

$$
\hat{Y}^{(k)}=F \hat{Y}^{(k-1)}
$$

with $\hat{Y}^{(0)}=Y$ and

$$
F=\left[\begin{array}{ccccccc}
0 & \cdots & \cdots & \cdots & \cdots & \cdots & 0 \\
a_{1} & 0 & \cdots & \cdots & \cdots & \cdots & 0 \\
a_{2} & a_{1} & 0 & \cdots & \cdots & \cdots & 0 \\
0 & a_{2} & a_{1} & 0 & \cdots & \cdots & 0 \\
0 & 0 & a_{2} & a_{1} & 0 & \cdots & 0 \\
\vdots & & \ddots & \ddots & \ddots & & \vdots \\
0 & \cdots & \cdots & 0 & a_{2} & a_{1} & 0
\end{array}\right]
$$

It is then clear that

$$
\hat{Y}^{(k)}=F^{k} Y
$$

The $N \times N$ matrix $F$ has its eigenvalues all equal to zero, and has the property $F^{N}=0$. The recurrent equation (6) is thus clearly stable. However, in simulations, for example, with $N=$ $50, a_{1}, a_{2}$ randomly drown within the interval $[0.5,1]$, and with $Y$ generated as a Gaussian noise, typically the simulated $\hat{Y}^{(k)}$ has some components increased several thousands times for some $k<N$. Of course, $\hat{Y}^{(k)}=0$ for $k \geq N$. The problem is that, for large $N$, some components of $\hat{Y}^{(k)}$ can become very large before the value of $k$ reaches $N$. Note that if $Y$ is by chance an eigenvector of $F$, then $\hat{Y}^{(k)}=0$ for all $k$, but this case does not often happen.

This "divergent" property observed by simulation is independent of the stability of (6), and also independent of the stability of the original AR equation (5). In fact, it does exist a positive definite matrix $P$ such that $\left(\hat{Y}^{(k)}\right)^{T} P \hat{Y}^{(k)}$ decreases with $k$ and it is true that $\hat{Y}^{(k)}$ tends to zero (it even reaches zero at $k=N$ ), but these facts do not prevent the rapid growth of some components of $\hat{Y}^{(k)}$ before $k=N$.

In order to better understand the problem, let us make some approximate analysis, since the accurate analysis seems extremely complicated. Define the $N \times N$ matrix

$$
J=\left[\begin{array}{ccccc}
0 & \cdots & \cdots & \cdots & 0 \\
1 & 0 & \cdots & \cdots & 0 \\
0 & 1 & 0 & \cdots & 0 \\
\vdots & \ddots & \ddots & & \vdots \\
0 & \cdots & 0 & 1 & 0
\end{array}\right]
$$

then

$$
\begin{aligned}
F & =a_{1} J+a_{2} J^{2} \\
& =J\left(a_{1} I_{N}+a_{2} J\right) \\
& =\left(a_{1} I_{N}+a_{2} J\right) J
\end{aligned}
$$

Therefore,

$$
\begin{aligned}
F^{k} & =J^{k}\left(a_{1} I_{N}+a_{2} J\right)^{k} \\
& =J^{k} \sum_{i=0}^{k} C_{k}^{i} a_{1}^{k-i} a_{2}^{i} J^{i}
\end{aligned}
$$

where

$$
C_{k}^{i}=\frac{k !}{(k-i) ! i !}
$$

The non zero entries of the matrix $F^{k}$ is thus expressed by $C_{k}^{i} a_{1}^{k-i} a_{2}^{i}$. Let us study some particular entry among them. For simplicity, assume $k$ is an even number. Let $k=2 m$ and consider $i=m$. It is of course assumed that $k+i=3 m<N$, otherwise $J^{k+i}=0$ and the corresponding entry would disappear from $F^{k}$. Then

$$
C_{2 m}^{m} a_{1}^{m} a_{2}^{m}=\frac{(2 m) !}{(m !)^{2}}\left(a_{1} a_{2}\right)^{m}
$$

For large $m$, the Stirling approximation formula

$$
n ! \approx \sqrt{2 \pi n}\left(\frac{n}{e}\right)^{n}
$$

can be applied, leading to

$$
\begin{aligned}
C_{2 m}^{m} a_{1}^{m} a_{2}^{m} & \approx \frac{\sqrt{2 \pi 2 m}\left(\frac{2 m}{e}\right)^{2 m}}{2 \pi m\left(\frac{m}{e}\right)^{2 m}}\left(a_{1} a_{2}\right)^{m} \\
& =\frac{(4 a b)^{m}}{\sqrt{\pi m}}
\end{aligned}
$$

Therefore, approximatively, if $|a b|>0.25$, then the entry $C_{2 m}^{m} a_{1}^{m} a_{2}^{m}$ will become large for large $m$.

This example shows the danger of the multiple steps prediction formula (3): even in the simple linear case, the computed results can become very large, and this bad behavior is independent of the stability of the considered AR model.

Remark that the matrix $F$ is not diagonalizable. If a matrix $M$ can be decomposed as $M=$ $U D U^{-1}$ with the diagonal matrix $D$ composed of the eigenvalues of $M$ and with some invertible matrix $U$, then $M^{k}=U D^{k} U^{-1}$. If $M$ has all its eigenvalues inside the unit circle, then $D^{k}$ does not grow with $k$, and there will be no problem to compute $M^{k}$. Therefore, it is the non diagonalizability and the large dimension of $F$ that have caused the "divergence" problem.

\section{METHOD 2}

In this section an alternative method is examined.

\subsection{Predictions and related errors}

Before formulating the second multiple steps prediction method, let us first play with iterations 
of the original NLARX model (1) to see how the measured outputs at different time instants are related by the model. For simplicity let us consider only the case of $n_{a}=2$ and forget the input which is not essential in the reasoning. In this case the original NLARX model (1) becomes

$$
y(t)=f[y(t-1), y(t-2)]+e(t)
$$

Apply the same equation to substitute $y(t-1)$ and $y(t-2)$ :

$$
\begin{aligned}
y(t)= & f\{f[y(t-2), y(t-3)]+e(t-1), \\
& f[y(t-3), y(t-4)]+e(t-2)\}+e(t)
\end{aligned}
$$

From this formula relating $y(t)$ to $y(t-2), y(t-$ $3), y(t-4)$ and to the errors $e(t), e(t-1), e(t-2)$, it seems natural to neglect the errors to predict $y(t)$ with the remaining parts of the equation:

$$
\begin{array}{r}
\hat{y}^{(2)}(t)=f\{f[y(t-2), y(t-3)], \\
f[y(t-3), y(t-4)]\}
\end{array}
$$

Indeed it coincides with the previously considered prediction formula (3). Its bad behavior has been shown in the previous section. Another explanation of this bad behavior is given in the following.

From the exact equation (8) to the approximative prediction (9), the only thing we have done is to neglect the errors $e(t), e(t-1), e(t-2)$. It is reasonable to neglect $e(t), e(t-1)$ if they are assumed to be independent of the measurements up to $y(t-2)$. However, after the measurement of $y(t-2)$, the error $e(t-2)$ can be deduced through $e(t-2)=y(t-2)-f[y(t-3), y(t-4)]$, it is thus not reasonable to neglect $e(t-2)$ for this prediction of $y(t)$. Therefore, as a correction to (9), the error $e(t-2)$ should be added to $f[y(t-3), y(t-4)]$, that exactly amounts to $y(t-2)$. Then the prediction should be computed as

$$
\hat{y}(t \mid t-2)=f\{f[y(t-2), y(t-3)], y(t-2)\}
$$

where the notation $\hat{y}(t \mid t-2)$ means the prediction of $y(t)$ from measured outputs up to $y(t-2)$. The point here is that, when a measured output is available, it should not be replaced by a predicted value.

Let us push a little bit further this example. Consider the 3 steps prediction of $y(t)$ that should be computed from measured outputs up to $y(t-3)$. Then in (10) the value of $y(t-2)$ is not available and must be replaced by a value predicted from earlier outputs. It is reasonable to replace it by $f[y(t-3), y(t-4)]$. The involved $y(t-3), y(t-4)$ should not be further substituted, following the rule of not substituting available outputs. After the substitution of the two occurrences of $y(t-2)$ in (10), the 3 steps ahead prediction of $y(t)$, noted as $\hat{y}(t \mid t-3)$, is then obtained.

The above prediction approach through substitutions of unknown values can be described in a recursive manner: for the $k$ steps ahead prediction of $y(t)$, namely $\hat{y}(t \mid t-k)$, starting by replace $y(t)$ with $f[y(t-1), y(t-2)]$. Then recursively replace each occurrence of $y(t-i), i=1,2, \ldots$, until $i=k$, since $y(t-k)$ is available and will not be further substituted. This procedure is formally stated by the recurrent equation

$$
\hat{y}(t \mid t-k)=f[\hat{y}(t-1 \mid t-k), \hat{y}(t-2 \mid t-k)]
$$

with the notation convention

$$
\hat{y}(t-k-i \mid t-k) \triangleq y(t-k-i) \text { for } i \geq 0
$$

It can be checked that, with this prediction formula, only the errors $e(t), e(t-1), \ldots, e(t-k+$ $1)$ are neglected. In the linear case, these errors appear linearly in the exact recurrent equation, and then $\hat{y}(t \mid t-k)$ coincides with the conditional mean $\mathbf{E}\left[y(t) \mid y(t-k), \ldots, y\left(t-k-n_{a}+1\right)\right]$ if the error sequence is a white noise. However, for general NLARX models, the multiple steps prediction $\hat{y}(t \mid t-k)$ does not coincide with the conditional mean.

More generally, for the NLARX model (1), the $k$ steps ahead prediction of the second method is given by the recurrent equation

$$
\begin{gathered}
\hat{y}(t \mid t-k)=f\left[\hat{y}(t-1 \mid t-k), \ldots, \hat{y}\left(t-n_{a} \mid t-k\right),\right. \\
\left.u\left(t-n_{k}\right), \ldots, u\left(t-n_{k}-n_{b}+1\right)\right]
\end{gathered}
$$

with

$$
\hat{y}(t-k-i \mid t-k) \triangleq y(t-k-i) \text { for } i \geq 0
$$

Notice that this $k$ steps prediction corresponds to the one step prediction (2) followed by $k-$ 1 steps noise-free simulation with the NLARX model. This method for multiple steps prediction is typically used in MPC. It can be better understood in a state space representation.

\subsection{State space representation of the second method}

Define

$$
\begin{gathered}
x(j)=\left[\begin{array}{c}
x_{1}(j) \\
\vdots \\
x_{n_{a}}(j)
\end{array}\right] \triangleq\left[\begin{array}{c}
\hat{y}(t-k+j \mid t-k) \\
\vdots \\
\hat{y}\left(t-k+j-n_{a}+1 \mid t-k\right)
\end{array}\right] \\
A \triangleq\left[\begin{array}{cccc}
0 & 0 & \cdots & 0 \\
1 & 0 & \cdots & 0 \\
\vdots & \ddots & \ddots & \vdots \\
0 & 0 & 1 & 0
\end{array}\right] \quad b \triangleq\left[\begin{array}{c}
1 \\
0 \\
\vdots \\
0
\end{array}\right] \quad c \triangleq\left[\begin{array}{llll}
1 & 0 & \cdots & 0
\end{array}\right]
\end{gathered}
$$

and

$$
\begin{aligned}
& \tilde{f}\left[x, u\left(t-n_{k}\right), \ldots, u\left(t-n_{k}-n_{b}+1\right)\right] \triangleq \\
& \quad f\left[x_{1}, \ldots, x_{n_{a}}, u\left(t-n_{k}\right), \ldots, u\left(t-n_{k}-n_{b}+1\right)\right]
\end{aligned}
$$

Then, the recurrent prediction formula (11) can be equivalently written as 

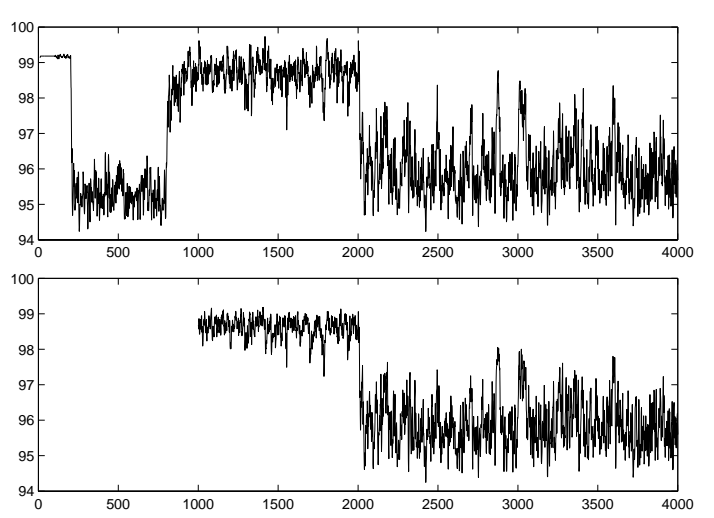

Figure 7. Ten steps (top) and one thousand steps (bottom) ahead prediction for the liquidsaturated steam heat exchanger data with the second method.

$$
\begin{aligned}
x(j) & =A x(j-1) \\
& +b \tilde{f}\left[x(j-1), u\left(t-n_{k}-k+j\right), \ldots,\right. \\
& \left.u\left(t-n_{k}-n_{b}+1-k+j\right)\right] \\
\hat{y}(t \mid t-k) & =c x(k)
\end{aligned}
$$

with the initial condition

$$
x(0)=\left[\begin{array}{c}
y(t-k) \\
\vdots \\
y\left(t-k-n_{a}+1\right)
\end{array}\right]
$$

Strictly speaking, equations (12)-(13) are not in the state space form, since past inputs are involved in the "state equation" (12). The dimension of the (quasi) state vector $x$ is $n_{a}$, independent of the data sample length $N$. Since $n_{a}$ is typically small, the "divergence" problem of the first method should not affect this second method.

Now let us illustrate the behavior of this prediction method with the example of the liquidsaturated steam heat exchanger which has put the first method into trouble. The results of 10 steps and 1000 steps ahead predictions given by this second method are plotted in figures 7. Even for the relatively long term (1000 steps) prediction, the result remains reasonable. Having shown the good prediction behavior in this example, let us remind that, in general, the behavior of the predictor depends also on the properties of the related system model.

\section{CONCLUSION}

There is no general simple way to compute optimal multiple steps prediction with NLARX models. For the purpose of engineering practice, two simple non optimal prediction methods for multiple steps prediction have been investigated in this paper. It is shown that cautions must be paid when using such methods, since their prediction behaviors may be radically different, depending on some detailed choice.

\section{REFERENCES}

Chen, Zengqiang, Jiangfeng He and Zhuzhi Yuan (1999). An adaptive identification and control scheme using radial basis function networks. Journal of Systems Engineering and Electronics 10(1), 54-61.

De Moor, B. L. R., Ed.) (1996). DaISy: Database for the Identification of Systems. Department of Electrical Engineering, ESAT/SISTA, K.U.Leuven, Belgium. URL: http://www.esat.kuleuven.ac.be/sista/daisy/.

Di Palma, F. and L. Magni (2004). A multimodel structure for model predictive control. Annual reviews in control 28, 47-52.

Doucet, Arnaud, de Freitas, Nando and Gordon, Neil, Eds.) (2001). Sequential Monte Carlo Methods in Practice. Statistics for Engineering and Information Science. SpringerVerlag. New York.

Goodwin, G. C. and R. L. Payne (1977). Dynamic system identification: Experiment design and data analysis. Academic Press. New York.

Jazwinski, A. H. (1970). Stochastic Processes and Filtering Theory. Vol. 64 of Mathematics in Science and Engineering. Academic Press. New York.

Juditsky, A., H. Hjalmarsson, A. Benveniste, B. Deylon, L. Ljung, J. Sjöberg and Q. Zhang (1995). Nonlinear black-box models in system identification: Mathematical foundations. Automatica 31(12), 1725-1750.

Levin, Asriel U. and Kumpati S. Narendra (1995). Identification using feedforward networks. Neural Computation 7(2), 349-357.

Ljung, L. (1999). System Identification: Theory for the User. Prentice Hall Information and System Sciences Series. Prentice Hall. Second edition.

Narendra, K.S. and K. Parthasarathy (1990). Identification and control of dynamical systems using neural networks. IEEE Trans. on Neural Networks $\mathbf{1} \mathbf{n}^{\circ} \mathbf{1}, 4-27$.

Principe, J. C., Ludong Wang and M. A. Motter (1998). Local dynamic modeling with self-organizing maps and applications to nonlinear system identification and control. Proceedings of the IEEE 86(11), 2240-58.

Söderström, Torsten and Petre Stoica (1989). System identification. Prentice-Hall International series in systems and control engineering. Prentice Hall.

Sjöberg, J., Q. Zhang, L. Ljung, A. Benveniste, B. Deylon, P-Y. Glorennec, H. Hjalmarsson and A. Juditsky (1995). Non-linear black-box modeling in system identification:a unified overview. Automatica 31(12), 1691-1724.

Walter, Eric and Luc Pronzato (1997). Identification of Parametric Models from Experimental Data. Communications and Control Engineering. Springer-Verlag. Berlin. 


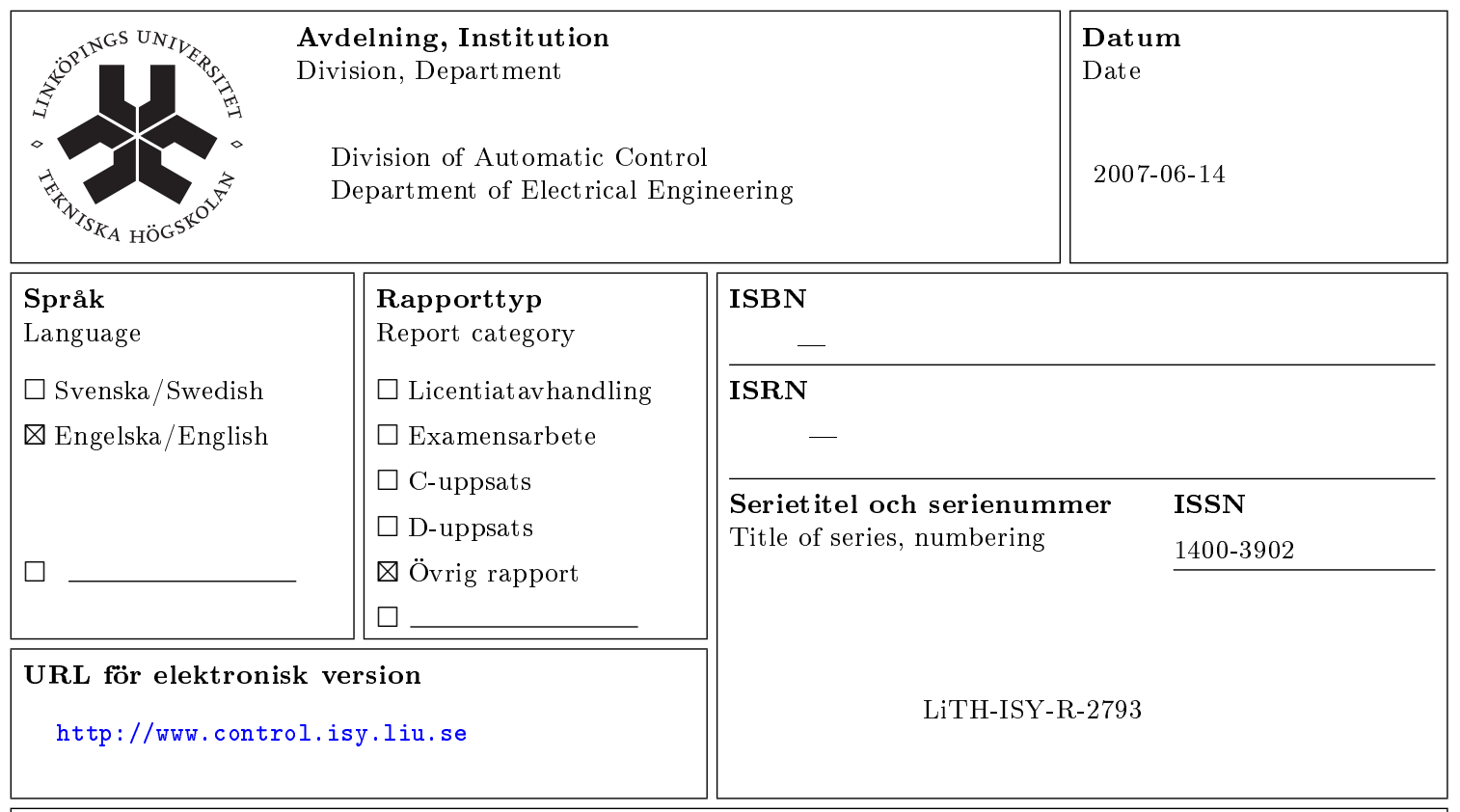

Titel Multiple steps prediction with nonlinear ARX models

Title

Författare Qinghua Zhang, Lennart Ljung

Author

\section{Sammanfattning}

Abstract

NLARX (NonLinear AutoRegresive with eXogenous inputs) models are frequently used in black-box nonlinear system identification. Though it is easy to make one step ahead prediction with such models, multiple steps prediction ids far from trivial. The main difficulty is that in general there is no easy way to compute the mathemaical expectation of an output conditioned by past measurements. An optimal solution would require intensive numerical computations related to nonlinear filtering. The purpose of this paper is to investigate simple non-optimal prediction merhods. It is shown that caution must be paid when using such methods, since the prediction behaviors may be radically different, depending on some detailed choices. 